\title{
Meron-Cluster Solution of Fermion Sign Problems
}

\author{
Shailesh Chandrasekharan ${ }^{\dagger}$ and Uwe-Jens Wiese ${ }^{\ddagger}$ \\ $\dagger$ Department of Physics, Box 90305, Duke University, Durham, NC 27708, U.S.A. \\ ¥ Center for Theoretical Physics, Massachusetts Institute of Technology, Cambridge, MA 02139, U.S.A.
}

(February 10, 1999)

\begin{abstract}
We present a general strategy to solve the notorious fermion sign problem using cluster algorithms. The method applies to various systems in the Hubbard model family as well as to relativistic fermions. Here it is illustrated for non-relativistic lattice fermions. A configuration of fermion world-lines is decomposed into clusters that contribute independently to the fermion permutation sign. A cluster whose flip changes the sign is referred to as a meron. Configurations containing meron-clusters contribute 0 to the path integral, while all other configurations contribute 1 . The cluster representation describes the partition function as a gas of clusters in the zero-meron sector.
\end{abstract}

02.70.Lq, 71.10.Fd, 05.50+q, 12.38.Gc

The numerical simulation of fermions is a notorious problem that hinders progress in understanding hightemperature superconductivity [1], QCD at non-zero chemical potential [2] and many other important problems in physics. One of the main problems originates from the minus-signs associated with Fermi statistics which prevent us from interpreting the Boltzmann factor in a fermionic path integral as a positive probability. When the sign of the Boltzmann factor is incorporated in measured observables, the fluctuations in the sign give rise to dramatic cancellations. Especially for large systems at low temperatures this leads to relative statistical errors that are exponentially large in both the volume and the inverse temperature. This makes it impossible in practice to study such systems with standard numerical methods. Here, for the first time, we completely eliminate a severe sign problem in the simulation of a nonrelativistic system of interacting lattice fermions using a cluster algorithm. The solution of the problem proceeds in two steps. The idea of the first step is to use cluster algorithm techniques to reduce the problem of canceling many contributions \pm 1 to the problem of averaging over non-negative contributions 0 and 1 . This step solves one half of the sign problem as discussed below. In large volumes and at small temperatures one still generates vanishing contributions to the average sign most of the time and very rarely one encounters a contribution 1 . In order to solve the other half of the problem a second step is necessary which guarantees that contributions 0 and 1 are generated with similar probabilities. The idea behind the second step is to include a Metropolis decision in the process of cluster decomposition. The two basic ideas behind our algorithm are general and apply to a variety of systems. In this paper, we illustrate our method for a simple model which serves as a testing ground for the new ideas.

Let us consider a fermionic path integral $Z_{f}=$ $\sum_{n} \operatorname{Sign}[n] \exp (-S[n])$ over configurations $n$ with a Boltzmann weight of $\operatorname{Sign}[n]= \pm 1$ and magnitude $\exp (-S[n])$. Here $S[n]$ is the action of a corre- sponding bosonic model with partition function $Z_{b}=$ $\sum_{n} \exp (-S[n])$. A fermionic observable $O[n]$ is obtained in a simulation of the bosonic ensemble as

$$
\langle O\rangle_{f}=\frac{1}{Z_{f}} \sum_{n} O[n] \operatorname{Sign}[n] \exp (-S[n])=\frac{\langle O \operatorname{Sign}\rangle}{\langle\operatorname{Sign}\rangle} .
$$

The average sign in the simulated bosonic ensemble is

$$
\langle\operatorname{Sign}\rangle=\frac{Z_{f}}{Z_{b}}=\exp (-\beta V \Delta f) .
$$

The last equality (valid for large $\beta V$ ) points to the heart of the sign problem. The expectation value of the sign is exponentially small in both the volume $V$ and the inverse temperature $\beta$ because the difference between the free energy densities $\Delta f=f_{f}-f_{b}$ of the fermionic and bosonic systems is necessarily positive. Even in an ideal simulation of the bosonic ensemble which generates $N$ completely uncorrelated configurations, the relative statistical error of the sign (again for large $\beta V$ ) is

$$
\frac{\Delta \operatorname{Sign}}{\langle\operatorname{Sign}\rangle}=\frac{\sqrt{\left\langle\operatorname{Sign}^{2}\right\rangle-\langle\operatorname{Sign}\rangle^{2}}}{\sqrt{N}\langle\operatorname{Sign}\rangle}=\frac{\exp (\beta V \Delta f)}{\sqrt{N}} .
$$

Here we have used $\operatorname{Sign}^{2}=1$. To determine the average sign with sufficient accuracy one needs to generate on the order of $N=\exp (2 \beta V \Delta f)$ configurations. For large volumes and small temperatures this is impossible in practice.

It is possible to solve one half of the problem if one can match all contributions -1 with 1 to give 0 , such that only a few unmatched contributions 1 remain. Then effectively Sign $=0,1$ and hence $\operatorname{Sign}^{2}=$ Sign. This reduces the relative error to

$$
\frac{\Delta \operatorname{Sign}}{\langle\operatorname{Sign}\rangle}=\frac{\sqrt{\langle\operatorname{Sign}\rangle-\langle\operatorname{Sign}\rangle^{2}}}{\sqrt{N^{\prime}}\langle\operatorname{Sign}\rangle}=\frac{\exp (\beta V \Delta f / 2)}{\sqrt{N^{\prime}}} .
$$

One gains an exponential factor in statistics, but one still needs to generate $N^{\prime}=\sqrt{N}=\exp (\beta V \Delta f)$ independent configurations in order to accurately determine the 
average sign [3]. This is because one generates exponentially many vanishing contributions before one encounters a contribution 1 .

In several cases cluster algorithms provide an explicit matching of contributions -1 and 1 using an improved estimator. Cluster algorithms are a very efficient tool to simulate quantum spin systems [4 [6]. In particular, the method can be implemented directly in the Euclidean time continuum [7]. The basic idea behind these algorithms is to decompose a configuration into $N_{C}$ clusters of spins which can be flipped independently. Averaging analytically over the $2^{N_{C}}$ configurations generated by the cluster flips, one can construct improved estimators for various physical quantities. As we will show, using an improved estimator for the fermion sign, cluster algorithms can solve the sign problem if the clusters contribute independently to the sign and a reference cluster orientation with a positive weight always exists. This means that the flip of any given cluster either changes the sign or not, independent of the orientation of all the other clusters. A cluster algorithm for lattice fermions was first presented in [8] with the hope of finding such an improved estimator. Unfortunately, in that algorithm the clusters do not affect the sign independent of one another. Still, cluster algorithms have been used for fermion models [9]. For systems with no severe sign problem these algorithms work much better than standard numerical methods, but they do not solve the fermion sign problem.

A solution to a sign problem using cluster algorithms was first found in a bosonic model with a complex action - the 2-d $O(3)$ model at vacuum angle $\theta=\pi[10]$. The cluster independence was achieved by constructing a non-standard action. In that model clusters whose flip changes the sign are half-instantons which are usually referred to as merons. In this paper we extend the meron-concept to fermionic models by demanding cluster independence. For non-relativistic spinless fermions hopping on a $d$-dimensional cubic lattice of size $V=L^{d}$ with periodic boundary conditions, this leads us to the Hamiltonian [1]

$$
H=\sum_{x, i}\left[-\frac{t}{2}\left(c_{x}^{+} c_{x+\hat{i}}+c_{x+\hat{i}}^{+} c_{x}\right)+U\left(n_{x}-\frac{1}{2}\right)\left(n_{x+\hat{i}}-\frac{1}{2}\right)\right],
$$

with $U \geq t>0$. Here $\hat{i}$ is a unit vector in the $i$-direction, $c_{x}^{+}$and $c_{x}$ are fermion creation and annihilation operators obeying the standard anticommutation relations and $n_{x}=c_{x}^{+} c_{x}$ is the occupation number of the lattice site $x$. Since $U>0$, two fermions or two holes on neighboring lattice sites repel each other, while a fermion and a hole attract one another. This is a simple example of a fermionic model for which the sign problem can be solved completely using a meron-cluster algorithm.

Let us now discuss our model and algorithm in more detail. Following [8] we introduce a space-time lattice with $2 d M$ time-slices and spacing $\varepsilon=\beta / M$ in the Euclidean time direction and we insert complete sets of occupation number $n(x, t)=0,1$ eigenstates at each timeslice to express the partition function as a path integral. The magnitude $\exp (-S[n])$ of the Boltzmann factor is a product of four-site interactions associated with spacetime plaquette configurations $[n(x, t), n(x+\hat{i}, t), n(x, t+$ $1), n(x+\hat{i}, t+1)]$. The sign factor $\operatorname{Sign}[n]= \pm 1$ has a topological meaning. The occupied sites form fermion world-lines which are closed in Euclidean time. Particles may be exchanged during their Euclidean time evolution and the fermion world-lines define a permutation of particles. According to the Pauli principle, Sign $[n]$ is just the sign of that permutation. In the following we restrict ourselves to $U=t$. Then the bosonic system without the sign factor is the antiferromagnetic spin $1 / 2$ quantum Heisenberg model, and $n(x, t)=0$ and 1 correspond to spin $-1 / 2$ and $1 / 2$, respectively. The staggered occupation (the analog of the staggered magnetization) $O[n]=\epsilon \sum_{x, t}(-1)^{x_{1}+x_{2}+\ldots+x_{d}}\left(n(x, t)-\frac{1}{2}\right)$, and the corresponding susceptibility $\chi=\left\langle O^{2} \operatorname{Sign}\right\rangle / \beta V\langle\operatorname{Sign}\rangle$ are important observables.

The algorithm decomposes a configuration into closed loops of lattice points which may be flipped independently. When a loop is flipped, the occupation numbers of all points on the loop are changed from 0 to 1 and vice versa. Each lattice point participates in two space-time plaquette interactions $[n(x, t), n(x+\hat{i}, t), n(x, t+1), n(x+$ $\hat{i}, t+1)]$. On each interaction plaquette the lattice points are connected in pairs and a sequence of connected points defines a loop-cluster. For space-time plaquette configurations $[0,0,0,0]$ and $[1,1,1,1]$ the lattice points are connected with their time-like neighbors, for configurations $[0,1,1,0]$ and $[1,0,0,1]$ they are connected with their space-like neighbors and for configurations $[0,1,0,1]$ and $[1,0,1,0]$ they are connected with their time-like neighbors with probability $p=2 /(1+\exp (\epsilon U))$ and with their space-like neighbors with probability $1-p$. After identifying the clusters, they are flipped independently with probability $1 / 2$.

A remarkable property of the cluster rules is that $\operatorname{Sign}[n]=\prod_{i=1}^{N_{C}} \operatorname{Sign}\left[C_{i}\right]$, where $C_{i}, i=1, \ldots, N_{C}$ denotes the oriented clusters in a configuration. By properly flipping the clusters, one can reach a reference configuration (the first configuration in figure 1) in which all even lattice sites are occupied and all odd sites are empty. In the reference orientation $\operatorname{Sign}\left[C_{i}\right]=1$. When the cluster is flipped, $\operatorname{Sign}\left[C_{i}\right]=1$ if $N_{w}+N_{h} / 2$ is odd and -1 otherwise. Here $N_{w}$ is the temporal cluster winding number and $N_{h}$ is the number of times the cluster hops to a neighboring lattice point. This relation follows directly from the fermionic anticommutation relations. Following [10], we refer to clusters whose flip changes the sign as merons. The flip of a meron-cluster permutes the fermions and changes the topology of the fermion world-lines. Since 
flipping all clusters does not change the fermion sign, the number of meron-clusters is always even. Two fermion configurations together with a meron-cluster are shown in figure 1 .
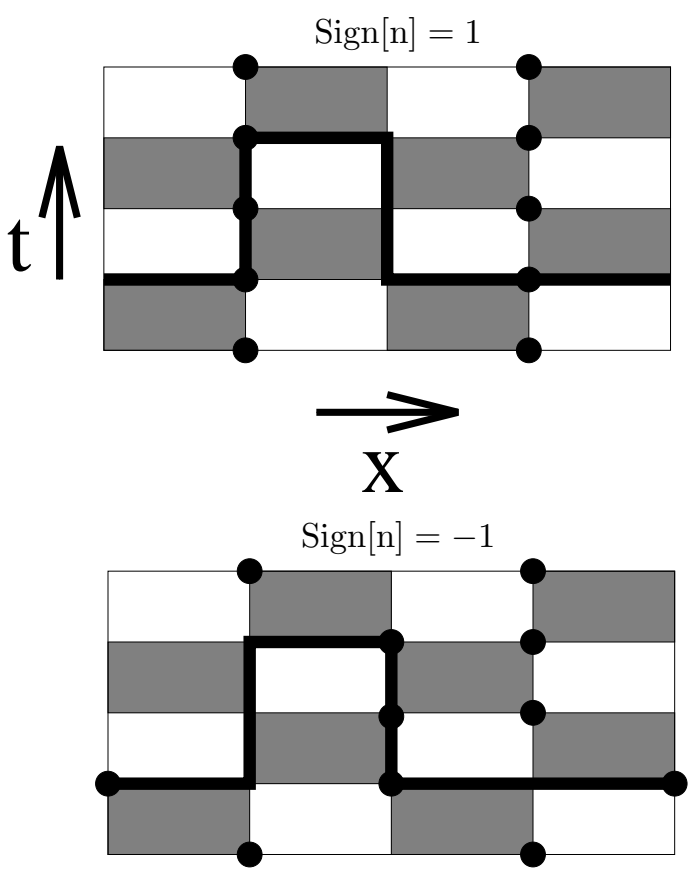

FIG. 1. Two configurations of fermion occupation numbers in $(1+1)$ dimensions. The shaded plaquettes carry the interaction. The dots represent occupied sites. In the second configuration two fermions interchange their positions. Flipping a meron-cluster (represented by the fat line) changes one configuration into the other and changes the fermion sign. The other clusters in the configurations are not shown.

The improved estimator for $\langle\operatorname{Sign}\rangle$ is the average over the $2^{N_{C}}$ configurations obtained from independently flipping the $N_{C}$ clusters in all possible ways. For configurations that contain merons the average sign is zero because flipping a single meron leads to a cancellation of signs \pm 1 . Only the configurations without merons contribute to $\langle\operatorname{Sign}\rangle$ and their contribution is always 1 . This solves one half of the sign problem as discussed before.

Let us now consider an improved estimator for $\left\langle O^{2} \mathrm{Sign}\right\rangle$ which is needed to determine the susceptibility $\chi$. The staggered occupation, $O[n]=\sum_{C} O_{C}$, is a sum of staggered occupations of the clusters, $O_{C}=$ $\epsilon \sum_{(x, t) \in C}(-1)^{x_{1}+x_{2}+\ldots+x_{d}}\left(n(x, t)-\frac{1}{2}\right)$. When a cluster is flipped, its staggered occupation changes sign. In a configuration without merons, where $\operatorname{Sign}[n]=1$ for all relative cluster flips, the average of $O[n]^{2} \operatorname{Sign}[n]$ over all $2^{N_{C}}$ configurations is $\sum_{C}\left|O_{C}\right|^{2}$. For configurations with two merons the average is $2\left|O_{C_{1}}\right|\left|O_{C_{2}}\right|$ where $C_{1}$ and $C_{2}$ are the two meron-clusters. Configurations with more than two merons do not contribute to $\left\langle O^{2} \operatorname{Sign}\right\rangle$. Thus, the improved estimator for the susceptibility is given by

$$
\chi=\frac{\left\langle\sum_{C}\left|O_{C}\right|^{2} \delta_{N, 0}+2\left|O_{C_{1}}\right|\left|O_{C_{2}}\right| \delta_{N, 2}\right\rangle}{V \beta\left\langle\delta_{N, 0}\right\rangle},
$$

where $N$ is the number of meron-clusters in a configuration. Hence, to determine $\chi$ one must sample the zeroand two-meron sectors only.

The probability to find a configuration without merons is exponentially small in the space-time volume since it is equal to 〈Sign〉. Thus, although we have increased the statistics tremendously with the improved estimators, without a second step one would still need an exponentially large statistics to accurately determine $\chi$. One goal of the second step is to eliminate all configurations with more than two merons. This enhances both the numerator and the denominator in eq.(6) by an exponentially large factor, but leaves their ratio unchanged. We start with an initial configuration with zero or two merons. For example, a completely occupied configuration has no merons. We then visit all plaquette interactions one after the other and choose new cluster connections between the four sites according to the cluster rules. If the new connection increases the number of merons beyond two, it is not accepted and the old connection is kept for that plaquette. To decide if the meron number changes, one needs to examine the clusters affected by the new connection. Although this requires a computational effort proportional to the cluster size (and hence to the physical correlation length) this is no problem, because one gains a factor that is exponentially large in the volume. The above procedure obeys detailed balance because configurations with more than two merons do not contribute to the observables we consider. Also, one can show that the algorithm is still ergodic. The simple reject step eliminates almost all configurations with weight 0 and is the essential step to solve the other half of the fermion sign problem.

Since for large space-time volumes the two-meron sector is much larger than the zero-meron sector, without further improvements one would still need statistics quadratic (but no longer exponential) in the space-time volume to accurately measure $\chi$. The remaining problem can be solved with a re-weighting technique similar to the one used in [10]. To enhance the zero-meron configurations in a controlled way, we introduce a trial probability $p_{t}(N)$ for each $N$-meron sector. We set $p_{t}(N)$ for $N>2$ to infinity and use it in a Metropolis accept-reject step for the newly proposed cluster connection on a specific plaquette interaction. A new connection that changes the meron number from $N$ to $N^{\prime}$ is accepted with probability $p=\min \left[1, p_{t}(N) / p_{t}\left(N^{\prime}\right)\right]$. In particular, configurations with $N^{\prime}>2$ are never generated because then $p_{t}\left(N^{\prime}\right)=\infty$ and $p=0$. After visiting all plaquette interactions, each cluster is flipped with probability $1 / 2$ which completes one update sweep. After re-weighting, the zero- and two-meron configurations appear with similar probabilities. This completes the second step in our 


\begin{tabular}{r|r|r|r|r|r}
\hline \hline \hline$L$ & $\beta U$ & $4 M$ & $\langle$ Sign $\rangle\left(\mathrm{A}_{1}\right)$ & $\chi\left(\mathrm{A}_{1}\right)$ & $\chi\left(\mathrm{A}_{2}\right)$ \\
\hline \hline 6 & 1.0 & 64 & $0.696(1)$ & $13.44(2)$ & $13.43(2)$ \\
\hline 8 & 1.0 & 64 & $0.536(3)$ & $13.53(3)$ & $13.52(3)$ \\
\hline 8 & 2.0 & 128 & $0.0164(5)$ & $199(3)$ & $203(2)$ \\
\hline 8 & 4.0 & 256 & $0.00051(7)$ & $690(100)$ & $729(9)$ \\
\hline 12 & 8.0 & 512 & - & - & $3090(130)$ \\
\hline \hline
\end{tabular}

TABLE I. Numerical results for $\langle$ Sign $\rangle$ and $\chi$ obtained with algorithm $A_{1}$ and $\chi$ obtained with algorithm $A_{2}$.

solution of the fermion sign problem. The re-weighting of the zero- and two-meron configurations is taken into account in the final expression for the susceptibility as

$$
\chi=\frac{\left\langle\sum_{C}\left|O_{C}\right|^{2} \delta_{N, 0} p_{t}(0)+2\left|O_{C_{1}}\right|\left|O_{C_{2}}\right| \delta_{N, 2} p_{t}(2)\right\rangle}{V \beta\left\langle\delta_{N, 0} p_{t}(0)\right\rangle} .
$$

We have implemented the meron cluster algorithm in $(2+1)$ dimensions and have tested it using exact diagonalization results on small lattices. Table 1 contains a comparison of results obtained with two algorithms using the same number of sweeps in both cases. The first algorithm $\left(A_{1}\right)$ has the improved estimators and solves one half of the sign problem. The second algorithm $\left(\mathrm{A}_{2}\right)$ has both the improved estimators and the additional Metropolis step and also solves the other half of the problem. The algorithm $\mathrm{A}_{2}$ is clearly superior once the average sign becomes small. In particular, we have applied $\mathrm{A}_{2}$ to systems of size $V=12^{2}$ at a low temperature $\beta U=8$. This is far beyond reach of standard fermion algorithms and even of the algorithm $A_{1}$. It should be noted that our model has a very severe sign problem which persists after integrating out the fermions even at half-filling.

Cluster representations in general and the meronconcept in particular are more than mere algorithmic tools. In fact, we have shown that the fermionic partition function can be expressed as a classical statistical mechanics system of clusters. The cluster formulation is a novel type of bosonization which works in any dimension. In this formulation the Pauli principle manifests itself by the vanishing Boltzmann weight of a configuration containing meron-clusters. If we ignore the fermion permutation sign, the theory describes a gas of merons and non-merons with a large configuration space. Including the sign factor forces even numbers of merons to be bound into non-merons. As a consequence, in agreement with the Pauli principle, the configuration space is very restricted. The merons allow us to simulate fermions with local bosonic variables. This is much more efficient than integrating out the fermions, which leads to non-local bosonic effective actions.

While the details of our algorithm are specific to the fermion model we have considered, the two basic ideas behind it are general and apply to a variety of models. They lead to a complete solution of the fermion sign problem for models of relativistic staggered fermions [11] as well as for non-relativistic fermions with spin. In applications of the meron-cluster algorithm to systems in the Hubbard model family we have so far not found high-temperature superconductivity. Meron-cluster algorithms are also applicable to quantum spin models in an arbitrary magnetic field for which a similar type of sign problem arises. Similarly, one can solve the sign problem resulting from a complex action in the 2-d $O(3)$ model at non-zero chemical potential or at non-zero vacuum angle $\theta$. The next challenge is to find applications of this method to QCD at non-zero baryon density. It seems likely that progress along the lines discussed here can be made in the quantum link D-theory formulation of the problem [12,13.

U.-J. W. likes to thank the physics department of Duke University where part of this work was done for its hospitality and the A. P. Sloan foundation for its support. This work is supported in part by funds provided by the U.S. Department of Energy (D.O.E.) under cooperative research agreements DE-FC02-94ER40818 and DE-FG0296ER40945.

[1] S. R. White et al., Phys. Rev. 40 (1989) 506; E. Dagotto et al., Phys. Rev. B41 (1990) 811.

[2] I. Barbour et al., Nucl. Phys. B (Proc. Suppl.) 60A (1998) 220 ;

M. Alford, A. Kapustin and F. Wilczek, Phys. Rev. D59 (1999) 054502.

[3] The fact that an improved estimator alone cannot solve the sign problem was pointed out to one of the authors by H. G. Evertz a long time ago.

[4] U.-J. Wiese and H.-P. Ying, Phys. Lett. A168 (1992) 143.

[5] H. G. Evertz, G. Lana and M. Marcu, Phys. Rev. Lett. 70 (1993) 875.

[6] U.-J. Wiese and H.-P. Ying, Z. Phys. B93 (1994) 147.

[7] B. B. Beard and U.-J. Wiese, Phys. Rev. Lett. 77 (1996) 5130.

[8] U.-J. Wiese, Phys. Lett. B311 (1993) 235.

[9] N. Kawashima, J. E. Gubernatis and H. G. Evertz, Phys. Rev. B50 (1994) 136;

B. Ammon et al., Phys. Rev. B58 (1998) 4304;

M. Brunner and A. Muramatsu, Phys. Rev. B58 (1998) R10100.

[10] W. Bietenholz, A. Pochinsky and U.-J. Wiese, Phys. Rev. Lett. 75 (1995) 4524.

[11] S. Chandrasekharan, J. Cox, K. Holland and U.-J. Wiese, hep-lat/9906021.

[12] S. Chandrasekharan and U.-J. Wiese, Nucl. Phys. B492 (1997) 455.

[13] R. Brower, S. Chandrasekharan and U.-J. Wiese, hepth/9704106, to appear in Phys. Rev. D. 